\title{
Longitudinal construct validity of the minimum data set health status index
}

Aaron Jones ${ }^{1}$, David Feeny ${ }^{2}$ and Andrew P. Costa ${ }^{1,3^{*}}$

\begin{abstract}
Background: The Minimum Data Set Health Status Index (MDS-HSI) is a generic, preference-based health-related quality of life (HRQOL) measure derived by mapping items from the Resident Assessment Instrument - Minimum Data Set (RAI-MDS) assessment onto the Health Utilities Index Mark 2 classification system. While the validity of the MDS-HSI has been examined in cross-sectional settings, the longitudinal validity has not been explored. The objective of this study was to investigate the longitudinal construct validity of the MDS-HSI in a home care population.

Methods: This study utilized a retrospective cohort of home care patients in the Hamilton-Niagara-Haldimand-Brant health region of Ontario, Canada with at least two RAI-MDS Home Care assessments between January 2010 and December 2014. Convergent validity was assessed by calculating Spearman rank correlations between the change in MDS-HSI and changes in six validated indices of health domains that can be calculated from the RAI-MDS assessment. Known-groups validity was investigated by fitting multivariable linear regression models to estimate the mean change in MDS-HSI associated with clinically important changes in the six health domain indices and 15 disease symptoms from the RAI-MDS Home Care assessment, controlling for age and sex.

Results: The cohort contained 25,182 patients with two RAI-MDS Home Care assessments. Spearman correlations between the MDS-HSI change and changes in the health domain indices were all statistically significant and in the hypothesized small to moderate range $[0.1<\rho<0.5]$. Clinically important changes in all of the health domain indices and 13 of the 15 disease symptoms were significantly associated with clinically important changes in the MDS-HSI.

Conclusions: The findings of this study support the longitudinal construct validity of the MDS-HSI in home care populations. In addition to evaluating changes in HRQOL among home care patients in clinical research, economic evaluation, and health technology assessment, the MDS-HSI may be used in system-level applications using routinely collected population-level data.
\end{abstract}

Keywords: Home care, Community care, Health-related quality of life, Longitudinal construct validity

\section{Background}

Generic, preference-based health-related quality of life (HRQOL) indices provide a multidimensional measure of various health domains and a single summary score of overall HRQOL and are essential for economic evaluation and health technology assessment [1]. Routine, repeated collection of preference-based HRQOL measures can enhance the capacity of health systems to assess how effectively they operate [2].

\footnotetext{
* Correspondence: acosta@mcmaster.ca

${ }^{1}$ Department of Health Research Methods, Evidence and Impact, McMaster University, 1280 Main St. W, Hamilton, ON L8S 4K1, Canada

${ }^{3}$ Department of Medicine, McMaster University, 1280 Main St. W, Hamilton L8S 4K1, ON, Canada

Full list of author information is available at the end of the article
}

The Resident Assessment Instrument - Minimum Data Set (RAI-MDS) is a comprehensive, standardized clinical assessment with variants used in nursing homes, complex continuing care, and home and community care populations in North America (i.e., Canada and many U.S. states), Europe, and Asia/Pacific Rim [3, 4]. The Minimum Data Set Health Status Index (MDS-HSI) is a generic, preference-based HRQOL measure derived by mapping items from the RAI-MDS assessment onto the Health Utilities Index Mark 2 classification system [5]. The MDS-HSI has been previously used in both cross-sectional and longitudinal studies [6, 7].

RAI-MDS assessment variants are mandated for use in multiple care settings in many jurisdictions. As the

(c) The Author(s). 2018 Open Access This article is distributed under the terms of the Creative Commons Attribution 4.0 International License (http://creativecommons.org/licenses/by/4.0/), which permits unrestricted use, distribution, and reproduction in any medium, provided you give appropriate credit to the original author(s) and the source, provide a link to the Creative Commons license, and indicate if changes were made. The Creative Commons Public Domain Dedication waiver (http://creativecommons.org/publicdomain/zero/1.0/) applies to the data made available in this article, unless otherwise stated. 
MDS-HSI is defined using items from the RAI-MDS assessment, the MDS-HSI can be calculated on population-based, routinely collected data in jurisdictions where collection of the RAI-MDS is standard practice. Thus the MDS-HSI could be used as a standard measure to compare regularly the HRQOL of patients across settings and jurisdictions, as well as within patient trajectories in the health system. While the validity of the MDS-HSI has been examined in cross-sectional contexts, the longitudinal construct validity of the measure has not been investigated $[8,9]$. Longitudinal construct validity, or responsiveness, refers to the ability of a measure to detect meaningful changes in a patient's condition [10].

The objective of this study was to examine the longitudinal construct validity of the MDS-HSI in a large home care population using convergent and known-groups approaches. We hypothesized that change in the MDS-HSI would exhibit small to moderate negative correlations with changes in six health domain indices. The correlations were hypothesized to be negative as the indices measure increasing impairment, and in the small to moderate range as they measure a single domain of health while the MDS-HSI is a multidimensional measure. We further hypothesized that clinically important improvements or declines in health domain indices and symptoms would be associated with corresponding clinically important improvements and declines in the MDS-HSI.

\section{Methods}

\section{Design and setting}

This study utilized a longitudinal, population-based, retrospective cohort of adult, home care patients in the Hamilton-Niagara-Haldimand-Brant region of Ontario, Canada. This health region is one of the largest health regions in Ontario with a population of over 1.5 million persons spread across urban, suburban, and rural locales. This health region contains over 10 municipalities with wide variations in population density, socioeconomic status, and access to tertiary care centers. Community-dwelling seniors receiving publicly-funded home care in Ontario are periodically assessed with the home care variant of the RAI-MDS, the RAI-MDS Home Care, as part of their routine care.

This study involved the retrospective analysis of routinely-collected health administrative data from secondary sources; there was no primary data collection. RAI-MDS assessments that are performed as part of routine care for home care patients in Ontario are stored in the Client and Health Information System (CHRIS), which is the health administrative database used by Ontario's publicly-funded home care agencies [11]. CHRIS additionally contains administrative data on home care episodes and billed services that can be individually linked to the clinical assessment records. CHRIS is regularly checked for validity and has been used extensively in research $[12,13]$. We utilized CHRIS records stored at the Health Data Library at McMaster University to create the retrospective cohort for this study. Ethics approval was obtained from the Hamilton Integrated Research Ethics Board.

\section{Participants}

An anonymized regional version of CHRIS was used to create a retrospective cohort of home care patients 19 years of age and older in the Hamilton-Niagara-Haldimand-Brant region with at least two RAI-MDS Home Care assessments. Administrative home care records were individually linked to clinical assessment records to identify adult patients who had a baseline assessment between January 1st, 2010 and December 31st, 2014, and a follow-up assessment between 3 and 18 months after the baseline assessment but before discharge from care. All patients 19 years of age and older with a valid baseline and follow-up assessment were included in the study.

\section{Measures}

All measures in the study were calculated from the RAI-MDS Home Care assessment. The RAI-MDS Home Care is a comprehensive clinical assessment that contains over 200 items in the domains of function, health, social support, and service use [3]. The internationally-developed assessment was created to identify multidimensional needs, aid in the development of appropriate care plans, and establish outcomes for tracking purposes. The RAI-MDS Home Care is used for clinical home care assessment in most Canadian provinces and territories, many U.S. states, and numerous countries in Europe and Asia/Pacific Rim. RAI-MDS Home Care assessments are typically performed in-person by registered nurses and take approximately 45 minutes to complete. RAI-MDS Home Care assessment data have previously been used in clinical and epidemiological research [14]. Average inter-assessor reliability weighed kappa scores for RAI-MDS Home Care items have been estimated at 0.74 for items that are shared with the RAI MDS 2.0 and 0.70 for stand-alone home care items [15]. Pearson correlation coefficients between functional and cognitive scales from the RAI-MDS Home Care assessment and gold standard scales have been calculated to be between 0.74 and 0.81 [16]. A comparison of the RAI-MDS Home Care assessment with the International Classification of Functioning, Disability, and Health has shown substantial overlap in content [17].

The primary measure of interest in this study was change in MDS-HSI between baseline and follow-up assessment. The MDS-HSI is a preference-based HRQOL measure based on the Health Utilities Index Mark 2 classification system and defined using items from the RAI-MDS Home Care assessment. The MDS-HSI is 
calculated using 31 elements of the RAI-MDS Home Care assessment to map a subject onto six health domains of the Health Utilities Index Mark 2 classification system (sensation, mobility, emotion, cognition, self-care, and pain). Published community Health Utilities Index Mark 2 preference weights are applied to produce scores [5]. Previous studies have supported the validity of the MDS-HSI in cross-sectional settings, finding an intraclass correlation coefficient (ICC) between the MDS-HSI and Health Utilities Index Mark 2 of 0.46 in a home care population, increasing to 0.63 with the $10 \%$ most discrepant scores removed [5, 8]. The MDS-HSI ranges from -0.03 to 1 , with 0 representing dead and 1 perfect health. As is true with the HUI2, MDS-HSI scores less than zero represent states worse than dead. A difference of 0.03 or more indicates a clearly clinically important difference $[18,19]$.

The measures used in this study to assess the convergent and known-groups validity of the MDS-HSI included six validated, ordinal indices of the health domains of function, cognition, health stability, pain, mood, and communication that are calculated from RAI-MDS Home Care assessment. The ADL Hierarchy Scale measures impairment in self-performance of the activities of daily living (ADL) tasks of dressing, eating, bathing and personal hygiene [20]. The ADL Hierarchy Scale ranges from 0 (Independent) to 6 (Total Dependence). The IADL Difficulty Scale measures difficulty in performing the instrumental activities of daily living (IADL) tasks of meal preparation, housework, and telephone use [21]. The IADL Difficulty Scale ranges from 0 (no difficulty in any of the three tasks) to 6 (great difficulty in all of the tasks). The Cognitive Performance Scale (CPS) measures impairment in memory and cognitive skills [22]. CPS ranges from 0 (intact cognition) to 6 (very severe impairment). The Changes in Health, End-Stage Disease, Signs, and Symptoms Scale (CHESS) measures health instability and risk of death [23]. The CHESS scale ranges from 0 (no health instability) to 5 (highly unstable health). The Pain Scale measures the frequency and intensity of pain and ranges from 1 (no pain) to 4 (excruciating pain) [24]. The Depression Rating Scale (DRS) measures the presence and severity of mood symptoms and ranges from 0 to 14 [25]. The Communication Scale measures impairment in expression or comprehension and ranges from 0 to 8 [26]. Neither the DRS nor the Communication Scale has descriptions for specific values of the index. Also used to assess known-groups validity were 15 disease symptoms recorded on the RAI-MDS Home Care assessment: angina, constipation, dizziness, edema, dyspnea, delusions, hallucinations, dysrhythmia, poor self-reported health, flare-up of chronic condition, disruptive pain, weight loss, one or fewer meals per day, insufficient fluid intake, and declining food/ liquid consumption. Age and sex were additional measures used as covariates in the linear regression analysis.

\section{Statistical analyses}

Longitudinal construct validity was examined using convergent and known-groups approaches. Convergent validity is an aspect of construct validity that can be generated by demonstrating that the measure of interest correlates with other measures as expected [27]. In a longitudinal setting, convergent validity can be investigated by examining the correlation between the change in the measure of interest and changes in other measures with which the measure of interest is expected to be associated. In this study, we assessed convergent validity by examining the correlations between the change in the MDS-HSI and the changes in six validated indices of individual health domains. Known-groups validity is another aspect of construct validity and can be generated by demonstrating that differences exist in the measure of interest between groups that are expected to score differently [27]. In a longitudinal context, known-groups validity can be investigated by examining average change in the measure of interest in groups that are known to have experienced a change in the underlying construct. In this study, we used changes in the six health domain indices and symptoms to define patients that were known to have experienced a clinically important change in their health status and examined whether the MDS-HSI indicated that there had been a clinically important change in HRQOL.

Convergent validity was assessed using correlational methods [28-30]. The association between the MDS-HSI change scores and changes in the health domain indices were examined with Spearman rank correlations $(\rho)$. The degree of the association was categorized according to Cohen: negligible $(\rho<0.1)$, small $(0.1 \leq \rho<0.3)$, moderate $(0.3 \leq \rho<0.5)$ and large $(\rho \geq 0.5)$ [31]. As the health domain indices represent single domains of health and the MDS-HSI is a multidimensional measure of HRQOL, small to moderate correlations were hypothesized. A negligible correlation would suggest that the MDS-HSI is not sensitive to change in the domain, while a large correlation would suggest that change in the domain may be inappropriately dominating change in the MDS-HSI. Given that the health domain indices measure increasing impairment on their individual domains, the correlations were hypothesized to be negative.

Known-groups validity was assessed using linear regression to estimate the mean change in MDS-HSI associated with a clinically important change in each of the health domains or symptoms while controlling for age and sex [30]. A clinically important change in any of the health 
domain indices or symptoms represents a change in the patient condition which would generally be expected to be associated with a clinically important change in HRQOL. Multivariable linear regression models were fit for each index and symptom with change in MDS-HSI as the dependent variable and change in the index or symptom as an independent variable. One-unit changes were taken to be clinically important for all indices except the depression rating scale, where, by convention, a two-unit change is considered clinically important. Minimal clinically important difference ratios were calculated by dividing the regression estimates of mean change in MSI-HSI by 0.03 . All models included age and sex as covariates with age treated as a linear continuous variable.

Linear regression was selected as the analysis method as the distribution of the change in MDS-HSI was expected to be reasonably close to the normal distribution (Appendix). Models were checked for multicollinearity and violations of model assumptions by examining variance inflation factors and residual and diagnostic plots. Cases with missing data were removed from the analysis. All analyses were performed using SAS 9.4 (SAS Institute, Cary NC).

\section{Sensitivity analysis}

Several sensitivity analyses were undertaken to investigate the robustness of results. These included restricting the follow-up assessment window to be between 6 and 12 months after the initial assessment rather than between 3 and 18 months, stratifying analyses by sex rather than adjusting for sex, using Pearson correlations rather than Spearman correlations, altering the formulation of the age covariate, and excluding age and sex from the models.

\section{Results}

\section{Descriptive characteristics}

There were 62,857 adult home care patients in the Hamilton-Niagara-Haldimand-Brant region with a baseline RAI-MDS Home Care assessment between January 1st, 2010 and December 31st, 2014 (Fig. 1). Of those patients, 25,187 received a follow-up RAI-MDS Home Care assessment between 90 and 540 days after the baseline assessment without being discharged from care between the assessments. Five cases $(0.02 \%)$ had missing data and were excluded from the analysis, reducing the final cohort to 25,182 .

The characteristics of the study cohort can be found in Table 1. The participants tended to be elderly $(78 \pm 14$ years) and nearly two-thirds were female (65.6\%). Just over one-third (35.1\%) lived alone. Significant impairments in the activities of daily living (45.4\%) and cognitive performance $(60.9 \%)$ were common, as well as mood symptoms (39.0\%) and a recent history of falls (41.5\%). A large majority of patients

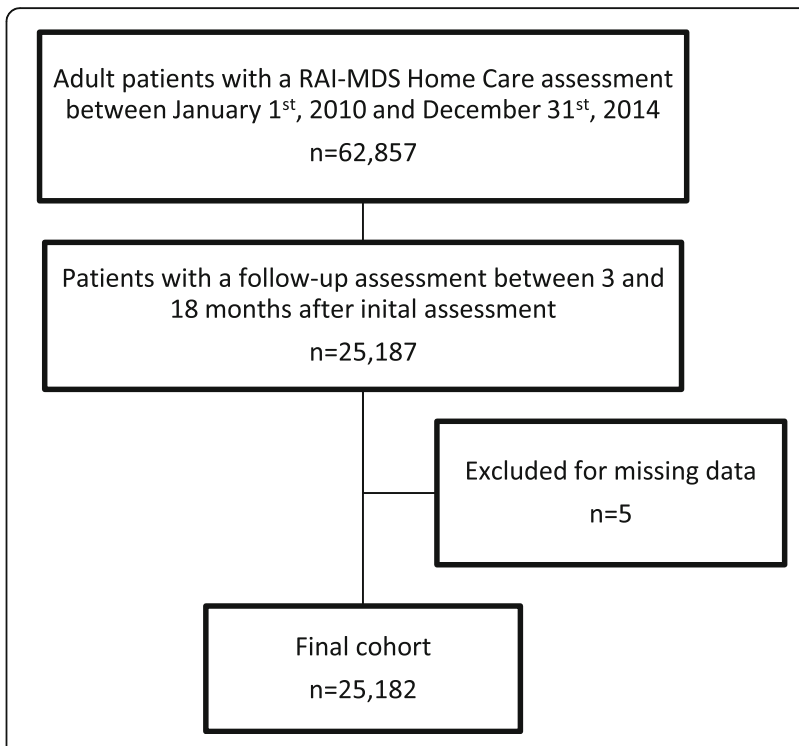

Fig. 1 Flow diagram of cohort creation

(83.6\%) had multiple chronic conditions. The mean baseline MDS-HSI was 0.50 , falling to 0.48 at follow-up. Between the baseline and follow-up assessments, 9872 (39.2\%) patients experienced a decline in MDS-HSI, 8767 (34.8\%) had no change, and 6543 (26.0\%) improved.

\section{Convergent validity}

Spearman rank correlation coefficients $(\rho)$ between the MDS-HSI change scores and health domain change scores are presented in Table 2. MDS-HSI change scores were significantly correlated with changes in each of the health domain indices, ranging from $-0.48[-0.49,-0.47]$ for the pain scale to $-0.21[-0.23,-0.20]$ for the communication scale. All correlations were negative and in the small to moderate range.

\section{Known-groups validity}

Results from the regression analysis can be found in Table 3. The mean change in MDS-HSI associated with clinically important changes in the health domain indices exceeded the minimal clinically important difference of 0.03 in each case. Minimal clinically important difference ratios varied from 1.27 for the communication scale to 3.04 for the pain scale.

Of the 15 symptoms examined, changes in 13 of them were associated with a clinically important change in the MDS-HSI. The greatest change was observed with the resolution or onset of pain intense enough to disrupt activities (minimal clinically important difference ratio 4.68), and the smallest change was associated with resolution or onset of dysrhythmia (minimal clinically important difference ratio 0.52). No variance inflation factors over 5 were observed and examination of residual and 
Table 1 Characteristics of participants in the study

\begin{tabular}{|c|c|}
\hline \multirow[t]{2}{*}{ Patient characteristics } & No. (\%) \\
\hline & $n=25,182$ \\
\hline \multicolumn{2}{|l|}{ Demographic Characteristics } \\
\hline Age (Mean $\pm S D)$ & $77.91 \pm(13.54)$ \\
\hline $\operatorname{Sex}(F)$ & $16,512(65.6 \%)$ \\
\hline Lives Alone & $8846(35.1 \%)$ \\
\hline \multicolumn{2}{|l|}{ Health Characteristics } \\
\hline ADL Impairment ${ }^{\mathrm{a}}$ & $11,436(45.4 \%)$ \\
\hline Cognitive Impairment $^{\mathrm{b}}$ & $15,431(60.9 \%)$ \\
\hline Dyspnea & $6440(25.6 \%)$ \\
\hline Poor Self-Reported Health & $4806(19.1 \%)$ \\
\hline Fall in last 90 Days & $10,447(41.5 \%)$ \\
\hline Mood Symptoms ${ }^{c}$ & $9831(39.0 \%)$ \\
\hline Wandering & $521(2.1 \%)$ \\
\hline Aggressive Behaviour $^{\mathrm{d}}$ & $1920(7.6 \%)$ \\
\hline Weight Loss ${ }^{\mathrm{e}}$ & $2098(8.3 \%)$ \\
\hline Number of Medications (Mean \pm SD) & $7.41 \pm(2.30)$ \\
\hline \multicolumn{2}{|l|}{ Informal Caregiver Status } \\
\hline Caregiver express distress $^{f}$ & $4568(18.1 \%)$ \\
\hline Information Care Hours per Day (Mean \pm SD) & $2.67 \pm(2.99)$ \\
\hline \multicolumn{2}{|l|}{ Diagnoses } \\
\hline Cardiovascular & $12,629(50.2 \%)$ \\
\hline Dementia & $5628(22.3 \%)$ \\
\hline Neurological & $2526(10.0 \%)$ \\
\hline Musculoskeletal & $16,662(66.2 \%)$ \\
\hline Psychiatric & $4591(18.2 \%)$ \\
\hline Cancer & $2818(11.2 \%)$ \\
\hline Diabetes & $6542(26.0 \%)$ \\
\hline COPD & $4379(17.4 \%)$ \\
\hline \multicolumn{2}{|l|}{ Count of Chronic Conditions ${ }^{9}$} \\
\hline 0 & $888(3.5 \%)$ \\
\hline 1 & $3249(12.9 \%)$ \\
\hline 2 & $5629(22.4 \%)$ \\
\hline 3 & $6395(25.4 \%)$ \\
\hline 4 & $4675(18.6 \%)$ \\
\hline 5 & $2639(10.5 \%)$ \\
\hline 6 & $1145(4.6 \%)$ \\
\hline $7+$ & $562(2.2 \%)$ \\
\hline
\end{tabular}

${ }^{\mathrm{a}}$ ADL Hierarchy Scale $>0$

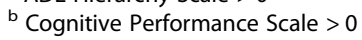

c Depression Rating Scale $>0$

${ }^{d}$ Verbal abuse, physical abuse, socially inappropriate behaviour, or resistance to care in last 3 days

e Unintended weight loss of $5 \%$ or more in the last 30 days or $10 \%$ or more in the last 180 days

${ }^{f}$ Caregiver expresses feelings of distress, anger, or depression

${ }^{9}$ Includes: Stroke, congestive heart failure, coronary artery disease, hypertension, dementia, Parkinson's disease, multiple sclerosis, arthritis, osteoporosis, psychiatric diagnosis, cancer, diabetes, emphysema, and renal failure
Table 2 Spearman correlation ( $\rho$ ) between change in health domain indices and change in MDS-HSI

\begin{tabular}{lll}
\hline Health Domain Index & $\rho(95 \% \mathrm{Cl})$ & $p$-value \\
\hline Change in ADL Hierarchy (Function) & $-0.375(-0.385,-0.364)$ & $<.0001$ \\
Change in IADL Difficulty (Function) & $-0.287(-0.298,-0.276)$ & $<.0001$ \\
Change in CPS (Cognition) & $-0.281(-0.292,-0.269)$ & $<.0001$ \\
Change in CHESS (Health Stability) & $-0.331(-0.342,-0.320)$ & $<.0001$ \\
Change in DRS (Mood) & $-0.380(-0.391,-0.370)$ & $<.0001$ \\
Change in Communication Scale & $-0.213(-0.225,-0.201)$ & $<.0001$ \\
Change in Pain Scale & $-0.482(-0.492,-0.473)$ & $<.0001$ \\
\hline
\end{tabular}

diagnostic plots did not indicate any substantial departures from model assumptions.

\section{Sensitivity analyses}

Restricting the population to only those with a valid follow-up assessment between 6 and 12 months after the initial assessment had no meaningful impact on the results (Tables 4 and 5). Stratifying results by sex produced very similar results for both males and females. Alternative formulations of age as a cubic smoothing spline or the inclusion of higher-order age polynomials had no significant impact on the model. The overall exclusion of age and sex from the models likewise had no significant impact.

\section{Discussion}

The longitudinal construct validity of an instrument is an important property to consider for any application that involves measuring change across multiple time points. This study investigated the longitudinal construct validity of the MDS-HSI with respect to changes in individual health domains and disease symptoms in adult, home care patients in Ontario, Canada. The authors are not aware of any previous study into the responsiveness of the MDS-HSI even though the measure has been used in longitudinal studies [6].

This investigation into the longitudinal convergent and known-groups validity of the MDS-HSI supports the responsiveness of the measure. Correlation between MDS-HSI change scores and change scores of the health domain indices were within expected ranges and indicated that the MDS-HSI is sensitive to changes in all of the domains examined. Regression analysis showed that clinically important changes in all of the indices and in 13 of the 15 symptoms were associated with clinically important changes in the MDS-HSI, indicating that the measure is able to detect differences in HRQOL among groups that are known to have changed.

Like other validated HRQOL measures, the MDS-HSI can be used in clinical research, economic evaluation, and health technology assessment. However, the ability of the MDS-HSI to be calculated from routinely-collected 
Table 3 Regression estimates of mean change in MDS-HSI by clinically important changes in indices and symptoms

\begin{tabular}{|c|c|c|c|}
\hline Measure & Mean change in MDS-HSI $(\beta)(95 \% \mathrm{Cl})^{\mathrm{a}}$ & $p$-value & MCID Ratio ${ }^{b}$ \\
\hline \multicolumn{4}{|l|}{ Health Domain Index } \\
\hline ADL Hierarchy (Function) & $-0.055(-0.057,-0.053)$ & $<.0001$ & 1.84 \\
\hline IADL Difficulty (Function) & $-0.045(-0.047,-0.043)$ & $<.0001$ & 1.50 \\
\hline CPS (Cognition) & $-0.065(-0.068,-0.062)$ & $<.0001$ & 2.16 \\
\hline CHESS (Health Stability) & $-0.050(-0.052,-0.049)$ & $<.0001$ & 1.68 \\
\hline DRS (two steps) (Mood) & $-0.066(-0.069,-0.064)$ & $<.0001$ & 2.21 \\
\hline Pain Scale & $-0.091(-0.093,-0.089)$ & $<.0001$ & 3.04 \\
\hline Communication Scale & $-0.038(-0.040,-0.036)$ & $<.0001$ & 1.27 \\
\hline \multicolumn{4}{|l|}{ Symptom } \\
\hline Angina & $-0.030(-0.040,-0.019)$ & $<.0001$ & 1.00 \\
\hline Constipation & $-0.057(-0.073,-0.042)$ & $<.0001$ & 1.91 \\
\hline Dizziness & $-0.039(-0.044,-0.033)$ & $<.0001$ & 1.28 \\
\hline Edema & $-0.028(-0.033,-0.022)$ & $<.0001$ & 0.92 \\
\hline Dyspnea & $-0.032(-0.038,-0.026)$ & $<.0001$ & 1.07 \\
\hline Delusions & $-0.081(-0.097,-0.066)$ & $<.0001$ & 2.71 \\
\hline Hallucinations & $-0.084(-0.097,-0.071)$ & $<.0001$ & 2.79 \\
\hline Dysrhythmia & $-0.016(-0.029,-0.002)$ & 0.022 & 0.52 \\
\hline Poor self-reported health & $-0.068(-0.075,-0.061)$ & $<.0001$ & 2.28 \\
\hline Flare-up of chronic condition & $-0.062(-0.070,-0.055)$ & $<.0001$ & 2.08 \\
\hline Disruptive Pain & $-0.140(-0.145,-0.135)$ & $<.0001$ & 4.68 \\
\hline Weight Loss & $-0.063(-0.070,-0.057)$ & $<.0001$ & 2.11 \\
\hline One or fewer meals per day & $-0.076(-0.087,-0.064)$ & $<.0001$ & 2.52 \\
\hline Insufficient fluid intake & $-0.086(-0.102,-0.069)$ & $<.0001$ & 2.86 \\
\hline Declining food/liquid consumption & $-0.110(-0.121,-0.099)$ & $<.0001$ & 3.67 \\
\hline
\end{tabular}

${ }^{a}$ Estimates correspond to an increase in the health domain index (increasing impairment) or new onset of a symptom

${ }^{\mathrm{b}} \mathrm{MCID}=\beta / 0.03$

population-level data suggests additional possibilities and applications. Typically, economic evaluation requires a prospective study incorporating an HRQOL survey. However, because the MDS-HSI can be calculated from data that is already stored in administrative health databases in many jurisdictions, there is the potential that population-level economic evaluation could be performed retrospectively. Additionally, regular measurement of HRQOL using a tool such as the MDS-HSI could produce benefits on both the patient and system level. For example, regular collection of a preference-based HRQOL measure for patients with chronic diseases would

Table 4 Sensitivity analysis of convergent validity results

\begin{tabular}{lllll}
\hline Health domain index & \multicolumn{2}{l}{ Spearman rank correlation coefficients $(\rho)$} & Male only & Female only \\
\cline { 2 - 5 } & Study population & 6 to 12 month follow-up window & $n=8670$ & $n=16,512$ \\
\hline Change in ADL Hierarchy (Function) & $n=25,182$ & $n=14,676$ & -0.385 & -0.368 \\
Change in IADL Difficulty (Function) & -0.375 & -0.386 & -0.262 & -0.304 \\
Change in CPS (Cognition) & -0.287 & -0.291 & -0.332 & -0.267 \\
Change in CHESS (Health Stability) & -0.331 & -0.302 & -0.371 & -0.330 \\
Change in DRS (Mood) & -0.380 & -0.322 & -0.217 & -0.210 \\
Change in Communication Scale & -0.213 & -0.372 & -0.476 & -0.486 \\
Change in Pain Scale & -0.482 & -0.229 & &
\end{tabular}

All correlations were statistically significant at $a=0.001$ 
Table 5 Sensitivity analysis of known-groups validity results

\begin{tabular}{|c|c|c|c|c|}
\hline \multirow[t]{3}{*}{ Measure } & \multicolumn{4}{|c|}{ Regression estimates ( $\beta$ ) of Mean Change in MDS-HSI } \\
\hline & Study population & 6 to 12 month follow-up window & Male only & Female only \\
\hline & $n=25,182$ & $n=14,676$ & $n=8670$ & $n=16,512$ \\
\hline \multicolumn{5}{|l|}{ Health domain index } \\
\hline ADL Hierarchy (Function) & -0.055 & -0.056 & -0.057 & -0.054 \\
\hline IADL Difficulty (Function) & -0.045 & -0.045 & -0.045 & -0.045 \\
\hline CPS (Cognition) & -0.065 & -0.068 & -0.068 & -0.063 \\
\hline CHESS (Health Stability) & -0.050 & -0.049 & -0.050 & -0.051 \\
\hline DRS (two steps) (Mood) & -0.066 & -0.065 & -0.072 & -0.064 \\
\hline Pain Scale & -0.091 & -0.091 & -0.086 & -0.094 \\
\hline Communication Scale & -0.038 & -0.041 & -0.038 & -0.038 \\
\hline \multicolumn{5}{|l|}{ Symptom } \\
\hline Angina & -0.030 & -0.024 & -0.039 & -0.025 \\
\hline Constipation & -0.057 & -0.061 & -0.055 & -0.058 \\
\hline Dizziness & -0.039 & -0.039 & -0.037 & -0.039 \\
\hline Edema & -0.028 & -0.026 & -0.022 & -0.030 \\
\hline Dyspnea & -0.032 & -0.028 & -0.036 & -0.030 \\
\hline Delusions & -0.081 & -0.081 & -0.087 & -0.078 \\
\hline Hallucinations & -0.084 & -0.083 & -0.089 & -0.081 \\
\hline Dysrhythmia & -0.016 & $-0.013^{*}$ & $-0.008^{*}$ & -0.020 \\
\hline Poor self-reported health & -0.068 & -0.061 & -0.076 & -0.064 \\
\hline Flare-up of chronic condition & -0.062 & -0.060 & -0.068 & -0.060 \\
\hline Disrupting Pain & -0.140 & -0.139 & -0.137 & -0.142 \\
\hline Weight Loss & -0.063 & -0.058 & -0.068 & -0.060 \\
\hline One or fewer meals per day & -0.076 & -0.071 & -0.070 & -0.078 \\
\hline Insufficient fluid intake & -0.086 & -0.083 & -0.096 & -0.081 \\
\hline Declining food/liquid consumption & -0.110 & -0.105 & -0.109 & -0.110 \\
\hline
\end{tabular}

* $p>0.05$

All other parameter estimates were statistically significant at $a=0.05$

allow clinicians and patients to develop care plans focused on improving the issues that are the most important to the patient. At the system level, regular collection of HRQOL data would enable a more general measurement of health system performance, providing a standard quality measurement across sectors and identifying key areas for improvement. The MDS-HSI would be an ideal instrument for regular measurement of HRQOL in care settings that routinely collect the RAI-MDS as no additional assessments would be required. As RAI-MDS assessment variants are used in numerous countries in North America, Europe, and Asia/Pacific Rim, the MDS-HSI could be used to measure and compare the HRQOL of millions of patients around the world with minimal change in practice.

\section{Limitations}

The limitations of this study include lack of access to an external gold standard criterion and uncertainty of the generalizability of the results outside of home care settings. Additional research should be conducted to continue to investigate the validity of the MDS-HSI, in particular, the comparability of results between different care settings.

\section{Conclusion}

The results of this study support the longitudinal construct validity of the MDS-HSI in home care populations. Analysis using convergent and known-groups approaches suggests that the MDS-HSI is responsive to changes in a broad array of health domains and disease symptoms. These findings support the usage of the measure to evaluate changes in HRQOL among home care patients in clinical research, economic evaluation, and health technology assessment. In addition, the calculability of the MDS-HSI from routinely collected data suggests other applications such as system-level performance measurement and use in retrospective studies. 


\section{Appendix}

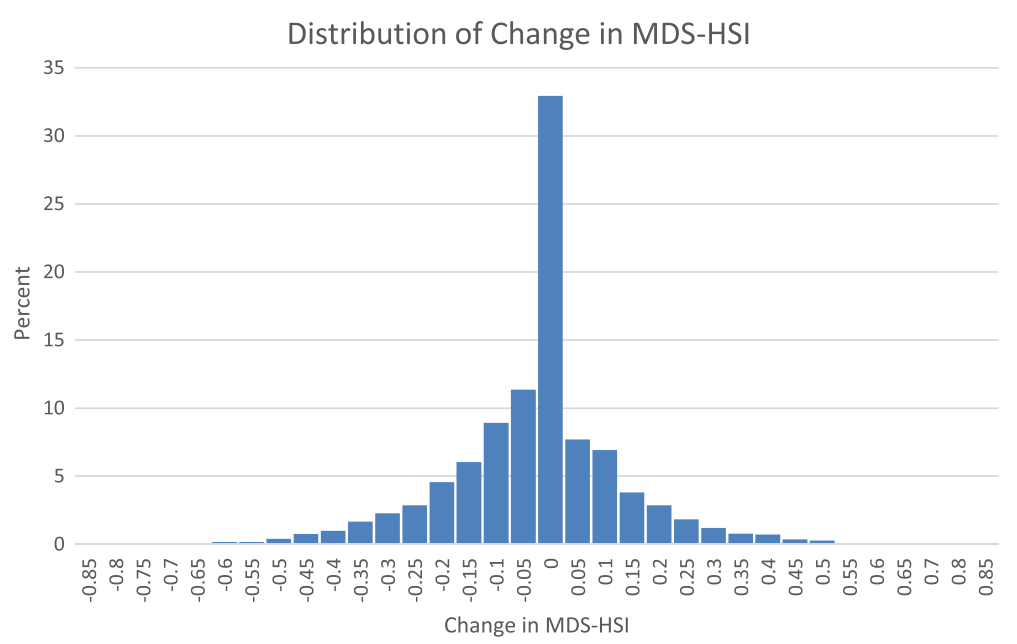

Fig. 2 Distribution of change in MDS-HSI

\section{Abbreviations}

ADL: Activities of daily living; CHESS: Changes in health, end-stage disease, signs and symptoms scale; CHRIS: Client and health information system; CPS: Cognitive performance scale; DRS: Depression rating scale; HRQOL: Health-related quality of life; IADL: Instrumental activities of daily living; ICC: Intraclass correlation coefficient; MDS-HSI: Minimum data set health status index; RAI-MDS: Resident assessment instrument - minimum data set

\section{Acknowledgments}

We would like to acknowledge the support of the Hamilton-NiagaraHaldimand-Brant Local Health Integration Network.

\section{Funding}

AC is supported by the Schlegel Chair in Clinical Epidemiology \& Aging, McMaster University. This study was funded by a grant from the Canadian Frailty Network (TG2015-15P). The opinions and conclusions expressed in this article are those of the authors and do not necessarily reflect the views of the contributing centers or funders.

\section{Availability of data and materials}

The datasets supporting the findings of this study are available upon reasonable request from the Canadian Institute of Health Information (ClHI).

\section{Authors' contributions}

$A C$ and $A J$ conceived the study and developed the design in consultation with the DF. AJ completed the analysis and drafted the initial manuscript. All authors contributed to the interpretation of data, the critical revision of the manuscript for important intellectual content, and approved the final version submitted for publication. AC and $\mathrm{AJ}$ take responsibility for the integrity of the data and the accuracy of the data analysis.

\section{Ethics approval and consent to participate}

Ethics approval was obtained from the Hamilton Integrated Research Ethics Board, 14-698-D.

\section{Competing interests}

It should be noted that DF has a proprietary interest in Health Utilities Incorporated, Dundas, Ontario, Canada. HUlnc distributes copyrighted Health Utilities Index (HUI) materials and provides methodological advice on the use of the HUI. AJ and AC have no competing interests.

\section{Publisher's Note}

Springer Nature remains neutral with regard to jurisdictional claims in published maps and institutional affiliations.

\section{Author details}

${ }^{1}$ Department of Health Research Methods, Evidence and Impact, McMaster University, 1280 Main St. W, Hamilton, ON L8S 4K1, Canada. ${ }^{2}$ Department of Economics, McMaster University, 1280 Main St. W, Hamilton, ON L8S 4M4, Canada. ${ }^{3}$ Department of Medicine, McMaster University, 1280 Main St. W, Hamilton L8S 4K1, ON, Canada.

Received: 28 August 2017 Accepted: 13 May 2018

Published online: 24 May 2018

\section{References}

1. Neumann PJ, Goldie SJ, Weinstein MC. Preference-based measures in economic evaluation in health care. Annu Rev Public Health. 2000;21:587-611.

2. Feeny D. Health-related quality-of-life data should be regarded as a vital sign. J Clin Epidemiol. 2013:66:706-9.

3. Hirdes JP, Ljunggren G, Morris JN, Frijters DH, Finne Soveri H, Gray L, et al. Reliability of the interRAI suite of assessment instruments: a 12-country study of an integrated health information system. BMC Health Serv Res. 2008;8:277.

4. Gray LC, Berg K, Fries BE, Henrard J-C, Hirdes JP, Steel K, et al. Sharing clinical information across care settings: the birth of an integrated assessment system. BMC Health Serv Res. 2009;9:71.

5. Wodchis WP, Hirdes JP, Feeny DH. Health-related quality of life measure based on the minimum data set. Int J Technol Assess Health Care. 2003;19:490-506.

6. Tarride J-E, Burke N, Leslie WD, Morin SN, Adachi JD, Papaioannou A, et al. Loss of health related quality of life following low-trauma fractures in the elderly. BMC Geriatr. 2016:16:84

7. Mondor L, Maxwell CJ, Bronskill SE, Gruneir A, Wodchis WP. The relative impact of chronic conditions and multimorbidity on health-related quality of life in Ontario long-stay home care clients. Qual Life Res. 2016;25:2619-32.

8. Wodchis WP, Maxwell CJ, Venturini A, Walker JD, Zhang J, Hogan DB, et al. Study of observed and self-reported HRQOL in older frail adults found group-level congruence and individual-level differences. J Clin Epidemiol. 2007:60:502-11.

9. Lam JMC, Wodchis WP. The relationship of 60 disease diagnoses and 15 conditions to preference-based health-related quality of life in Ontario hospital-based long-term care residents. Med Care. 2010;48:380-7.

10. Revicki DA, Osoba D, Fairclough D, Barofsky I, Berzon R, Leidy NK, et al. Recommendations on health-related quality of life research to support 
labeling and promotional claims in the United States. Qual Life Res. 2000;9: 887-900.

11. Zvaniga C. Chris: a community health care success story. Toronto: BPS Books; 2011.

12. Seow H, Brazil K, Sussman J, Pereira J, Marshall D, Austin PC, et al. Impact of community based, specialist palliative care teams on hospitalisations and emergency department visits late in life and hospital deaths: a pooled analysis. BMJ. 2014;348:93496

13. Seow H, Barbera L, Howell D, Dy SM. Using more end-of-life homecare services is associated with using fewer acute care services: a populationbased cohort study. Med Care. 2010;48:118-24.

14. Fialová D, Topinková E, Gambassi G, Finne-Soveri H, Jónsson PV, Carpenter I, et al. Potentially inappropriate medication use among elderly home care patients in Europe. JAMA. 2005;293:1348-58.

15. Morris JN, Fries BE, Steel K, Ikegami N, Bernabei R, Carpenter Gl, et al. Comprehensive clinical assessment in community setting: applicability of the MDS-HC. J Am Geriatr Soc. 1997:45:1017-24.

16. Landi F, Tua E, Onder G, Carrara B, Sgadari A, Rinaldi C, et al. Minimum data set for home care: a valid instrument to assess frail older people living in the community. Med Care. 2000;38:1184-90.

17. Berg K, Finne-Soveri H, Gray L, Henrard JC, Hirdes J, Ikegami N, et al. Relationship between interRAI HC and the ICF: opportunity for operationalizing the ICF. BMC Health Serv Res. 2009;9:47.

18. Horsman J, Furlong W, Feeny D, Torrance G. The health utilities index (HUIø): concepts, measurement properties and applications. Health Qual Life Outcomes. 2003;1:54.

19. Drummond M. Introducing economic and quality of life measurements into clinical studies. Ann Med. 2001;33:344-9.

20. Morris JN, Fries BE, Morris SA. Scaling ADLs within the MDS. J Gerontol A Biol Sci Med Sci. 1999;54:M546-53.

21. Poss JW, Hirdes JP, Fries BE, McKillop I, Chase M. Validation of Resource Utilization Groups version III for Home Care (RUG-III/HC): evidence from a Canadian home care jurisdiction. Med Care. 2008:46:380-7.

22. Hartmaier SL, Sloane PD, Guess HA, Koch GG, Mitchell CM, Phillips CD. Validation of the minimum data set cognitive performance scale: agreement with the mini-mental state examination. J Gerontol A Biol Sci Med Sci. 1995;50:M128-33.

23. Hirdes JP, Frijters DH, Teare GF. The MDS-CHESS scale: a new measure to predict mortality in institutionalized older people. J Am Geriatr Soc. 2003;51:96-100

24. Fries BE, Simon SE, Morris JN, Flodstrom C, Bookstein FL. Pain in US nursing homes validating a pain scale for the minimum data set. The Gerontologist. 2001;41:173-9.

25. Burrows AB, Morris JN, Simon SE, Hirdes JP, Phillips C. Development of a minimum data set-based depression rating scale for use in nursing homes. Age Ageing. 2000;29:165-72.

26. Frederiksen $K$, Tariot P, De Jonghe E. Minimum Data Set Plus (MDS+) scores compared with scores from five rating scales. J Am Geriatr Soc. 1996;44:305-9.

27. Fayers PM, Machin D. Quality of life: the assessment, analysis and interpretation of patient-reported outcomes. 2nd ed. Chichester: Wiley; 2013.

28. Stratford PW, Binkley JM, Riddle DL. Health status measures: strategies and analytic methods for assessing change scores. Phys Ther. 1996;76:1109-23.

29. Terwee CB, Dekker FW, Wiersinga WM, Prummel MF, Bossuyt PMM. On assessing responsiveness of health-related quality of life instruments: guidelines for instrument evaluation. Qual Life Res. 2003;12:349-62.

30. Husted JA, Cook RJ, Farewell VT, Gladman DD. Methods for assessing responsiveness. J Clin Epidemiol. 2000;53:459-68.

31. Cohen J. Statistical power analysis for the behavioral sciences. 2nd ed. Hillsdale: Lawrence Earlbaum Associates; 1988.

\section{Ready to submit your research? Choose BMC and benefit from:}

- fast, convenient online submission

- thorough peer review by experienced researchers in your field

- rapid publication on acceptance

- support for research data, including large and complex data types

- gold Open Access which fosters wider collaboration and increased citations

- maximum visibility for your research: over $100 \mathrm{M}$ website views per year

At BMC, research is always in progress.

Learn more biomedcentral.com/submissions 